\title{
Ground-state fidelity of the spin-1 Heisenberg chain with single ion anisotropy: quantum renormalization group and exact diagonalization approaches
}

\author{
A. Langari, ${ }^{1,2,3}$ F. Pollmann, ${ }^{3}$ and M. Siahatgar ${ }^{3}$ \\ ${ }^{1}$ Department of Physics, Sharif University of Technology, P.O.Box 11155-9161, Tehran, Iran \\ ${ }^{2}$ Center of excellence in Complex Systems and Condensed Matter (CSCM), \\ Sharif University of Technology, Tehran 1458889694, Iran \\ ${ }^{3}$ Max-Planck-Institut für Physik komplexer Systeme, 01187 Dresden, Germany*
}

(Dated: November 1, 2018)

\begin{abstract}
We study the phase diagram of the anisotropic spin-1 Heisenberg chain with single ion anisotropy (D) using a ground-state fidelity approach. The ground-state fidelity and its corresponding susceptibility are calculated within the quantum renormalization group scheme where we obtained the renormalization of fidelity preventing to calculate the ground state. Using this approach, the phase boundaries between the antiferromagnetic Néel, Haldane and large- $D$ phases are obtained for the whole phase diagram, which justifies the application of quantum renormalization group to trace the symmetery protected topological phases. In addition, we present numerical exact diagonalization (Lanczos) results in, which we employ a recently introduced non-local order parameter to locate the transition from Haldane to large- $D$ phase accurately.

PACS numbers: 75.10.Pq, 64.60.ae, 64.70.Tg, 03.67.-a
\end{abstract}

\section{INTRODUCTION}

A major challenge in the physics of correlated electrons is to understand quantum phases and the transitions between different phases. Quantum spin chains have proven to be extremely useful model systems as these relatively simple models can exhibit very complex phase diagrams $\stackrel{\underline{1}}{\underline{1}}$ The different phases are usually characterized by spontaneous symmetry breaking and can be understood in the framework of Landau's

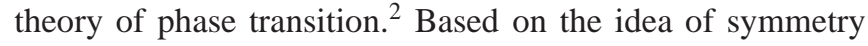
breaking, local order parameters can be defined that distinguish the different phases. Over the past few decades, several phases have been discovered, which do not break any symmetry and thus cannot be understood using local order parameters. Such phases are known as "topological phases". A well known example of a topological phase is the Haldane phase in spin-chains with odd integer spin ${ }^{3.4}$ The Haldane phase is an example of a so-called symmetry protected topological phase (SPTP). In a series of works, it has been shown that SPTP's can be completely characterized using cohomology theory $\underline{\underline{5}-9}$ In the case of the Haldane phase any of the following symmetries is sufficient to protect the phase: the dihedral group of $\pi$-rotations about two orthogonal axes, time-reversal symmetry, or bond centered inversion symmetry.

Transitions between different quantum phases are accompanied by a qualitative change of the ground state, driven by quantum fluctuations as a control parameter in the Hamiltonian is tuned. Apart from few exactly solvable models (e.g., the transverse field Ising model), the precise determination of the quantum critical point is a very challenging task - even if a local order parameter changes at the transition. If no order parameter exists to distinguish the phases, the task is even more difficult. Generically, phase transitions are characterized by detecting a non-analytic behavior in some physical properties of the system. Intensive investigations have been devoted to characterize quantum phase transition using quantum information concepts $\stackrel{10,11}{\longleftarrow}$ It turned out that the ground-state fidelity is a particular powerful quantity to identify a quantum phase transition, irrespective of the existence of global symmetry breaking. Thus it is very useful when studying topological phase transitions. $\stackrel{12.13}{\underline{1}}$ An essential change of the ground state at the quantum critical point leads to an abrupt drop of ground state fidelity accompanied by a divergent peak of its corresponding susceptibility $\underline{\underline{14}}$

Recently it has been proposed to use the "quantum renormalization group" (QRG) method to obtain the quantum-information related properties of strongly correlated systems $.15-17$ The QRG is a technique based on coarse graining to the most important degrees of freedom in the low energy spectrum $\stackrel{18,19}{ }$ It has been shown that the ground-state fidelity can be obtained in terms of QRG procedure without the actual need to obtain the ground state 20,21 Motivated by this approach to investigate quantum critical behaviors in terms of QRG for ground-state fidelity, it is very interesting to apply this technique to topological quantum phase transitions.

In this paper, we consider the anisotropic spin-1 Heisenberg chain with single ion anisotropy. Besides various symmetry broken phases, this model has two phases, which do not break any symmetry: The Haldane phase, i.e., a SPTP is found around the isotropic point, and in the presence of a strong ion anisotropy the system is in the so-called large$D$ phase (which is adiabatically connected to a simple site factorized state). We apply the QRG algorithm to calculate the ground-state fidelity and its corresponding susceptibility. Moreover, we present exact diagonalization (Lanczos) results on finite clusters, which are compared to the QRG results. We calculate non-local order parameters based on the inversion symmetry (which protects the Haldane phase) to obtain a precise determination of quantum critical point on finite clusters. Here we use the fact, that the expectation value of any member of permutation operator on a closed ring is negative 


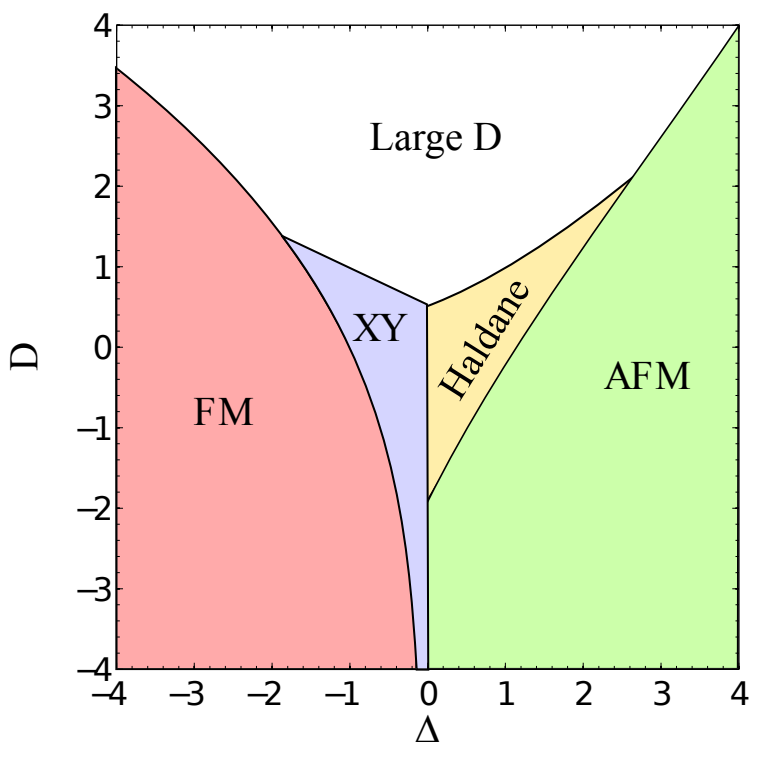

FIG. 1. (color online) A sketch of the spin-1 phase diagram based on results from Ref. 22 .

in the Haldane phase while it becomes positive in the large- $D$ phase $\frac{7}{\underline{7}}$

The remainder of this paper is organized as follows: In the next section we introduce the model and summarize some known results. In Sec. [II] we explain how to use the QRG approach to obtain the ground-state fidelity and analyze its behavior close to quantum critical points, which leads to the phase diagram of the spin-1 chain. We present exact diagonalization results in Sec. IV] where we also introduce the parity order parameter, which changes sign at the Gaussian critical point. Finally, we present a summary and we conclude our results in Sec. D.

\section{MODEL}

We focus on the anisotropic spin-1 Heisenberg chain with a single ion anisotropy $(D)$. The model Hamiltonian is given by

$$
H=J \sum_{i=1}^{N}\left[S_{i}^{x} S_{i+1}^{x}+S_{i}^{y} S_{i+1}^{y}+\Delta S_{i}^{z} S_{i+1}^{z}+D\left(S_{i}^{z}\right)^{2}\right],
$$

where $J$ is the antiferromagnetic exchange coupling, $\Delta$ is the easy axis anisotropy and $D$ represents the single-ion anisotropy parameter. The phase diagram of Hamiltonian Eq. (1) is well established ${ }^{22-28}$ and sketched in Fig. 1. It contains a gapless XY, a ferromagnetic (FM) and an anti ferromagnetic (AF) phase. The increase of $D$ drives the ground state of model from Haldane to large- $D$ phase through a Gaussian transition ${ }^{24}$ The lack of a local order parameter for the Gaussian transition and its topological nature make the study very challenging. The phase diagram has been studied using a density matrix renormalization group (DMRG) computation of ground-state fidelity for both the isotropic 29 and anisotropic ${ }^{26}$ chain. It was found for the isotropic model $(\Delta=$ 1) that the fidelity susceptibility diverges at the phase transitions out of the Haldane phase according to scaling relations defined in Ref. 27. However, the fidelity susceptibility fails to detect the Gaussian transition for an anisotropy parameter of $\Delta=0.5$. The scaling analysis performed in Ref. 26 found a critical exponent for the correlation length of $\nu \simeq 1.51$. Extensive DMRG simulations ${ }^{28}$, in which the length of chain goes to $N=10000$ and the number of kept states can reaches $m=1000$, were implemented to study the quantum critical properties of spin-1 chain accurately. However, the exponent reported in the latter work (Ref. 28) is $\nu \simeq 2.387(5)$ for $\Delta=0.5$. This discrepancy represents the non-local nature of strong correlation in the ground state of model specially close to quantum critical point, which makes the precise determination of quantum critical properties a challenging task.

\section{QUANTUM RENORMALIZATION GROUP APPROACH AND GROUND-STATE FIDELITY}

The QRG proceeds by keeping the most important degrees of freedom while integrating out the rest within an iterative procedure. As a result, the original Hamiltonian is mapped into a renormalized Hamiltonian defined by the set of renormalized couplings. The renormalization of couplings generates the flow of couplings, which describes the quantum phase diagram in addition to the ground state properties of the model.

The standard QRG prescription, which is implemented in this work, is based on Kaddanoff block renormalization group $19,30-34$ The algorithm of this procedure can be expressed in the following steps: (i) The lattice is decomposed into isolated blocks (see Fig. 2) where the Hamiltonian is written as a sum of isolated block Hamiltonian $\left(H^{B}\right)$ and interblock interactions $\left(H^{B B}\right)$, i.e., $H=H^{B}+H^{B B}$. (ii) The block Hamiltonian is diagonalized exactly and some of the low-lying energy eigenstates of each block are kept to build up an embedding (projection) operator $(T)$, representing the most important subspace of the original Hilbert space $(\mathcal{H})$. (iii) The original Hamiltonian $(H)$ is mapped into the renormalized (effective) Hamiltonian $\left(H^{\prime}\right)$ utilizing the embedding operator, i.e.,

$$
H^{\prime}=T^{\dagger} H T
$$

The renormalized Hamiltonian, which is supposed to be self similar to the original one, defines the renormalization of coupling constants (QRG-flow).

In our implementation of the QRG procedure, the model Eq. (1) is decomposed into individual blocks of three spins, see Fig. 2, where the intra-block and inter-block Hamiltonians 


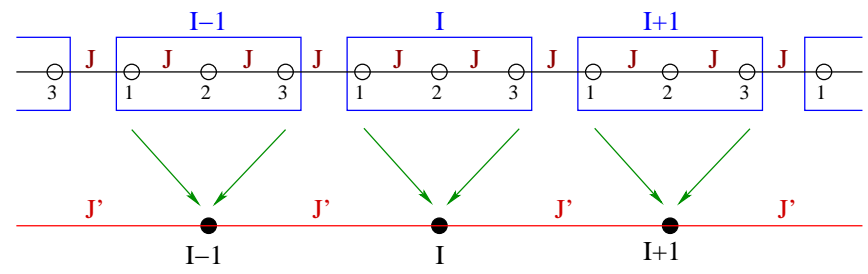

FIG. 2. (color online) Decomposition of the chain into blocks. Each block will be represented by an effective spin (filled-circle) after the renormalization with renormalized interactions.

are given by the following relations

$$
\begin{gathered}
H^{B}=\sum_{I=1}^{N / 3} h_{I}^{B}, \quad H^{B B}=\sum_{I=1}^{N / 3} h_{I, I+1}^{B B}, \\
h_{I}^{B}=J\left[\sum_{j=1}^{2}\left(S_{I, j}^{x} S_{I, j+1}^{x}+S_{I, j}^{y} S_{I, j+1}^{y}+\Delta S_{I, j}^{z} S_{I, j+1}^{z}\right)\right. \\
\left.+D \sum_{j=1}^{3}\left(S_{I, j}^{z}\right)^{2}\right], \\
h_{I, I+1}^{B B}=J\left(S_{I, 3}^{x} S_{I+1,1}^{x}+S_{I, 3}^{y} S_{I+1,1}^{y}+\Delta S_{I, 3}^{z} S_{I+1,1}^{z}\right) .
\end{gathered}
$$

Here $S_{I, j}^{\alpha}$ denotes the $\alpha$-component of the $j$-th spin in block $I$. The energy eigenstates of $h_{I}^{B}$ are calculated exactly and the three lowest eigenvectors are denoted by $\left|\phi_{0}\right\rangle$ and $\left|\phi_{ \pm}\right\rangle$ with the corresponding eigenvalues $E_{0}$ and $E_{1}$, respectively. The first excited state energy $\left(E_{1}\right)$ is doubly degenerate (corresponding to $S^{z}= \pm 1$ ) Accordingly, the embedding operator for each block is constructed by

$$
T_{I}=\left|\phi_{+}\right\rangle\left\langle+1|+| \phi_{0}\right\rangle\left\langle 0|+| \phi_{-}\right\rangle\langle-1|,
$$

where $| \pm 1\rangle,|0\rangle$ are the base kets for the renormalized Hilbert space of each block. The projection of the original Hamiltonian to the renormalized Hilbert space of the whole system is done by the global embedding operator $\left(T=\otimes_{I=1}^{N / 3} T_{I}\right)$ as defined in Eq. (2). The renormalized Hamiltonian $\left(H^{\prime}\right)$ is akin to the original one, Eq. (1), replacing the couplings with the following renormalized ones, i.e.,

$$
J^{\prime} \equiv J^{\prime}(J, D, \Delta), D^{\prime} \equiv D^{\prime}(D, \Delta), \Delta^{\prime} \equiv \Delta^{\prime}(D, \Delta) .
$$

The explicit form of renormalized couplings in terms of the original ones and some details of the renormalization procedure are presented in Appendix $\mathrm{A}$. The ground state properties of the model can be extracted from the QRG-flow or equivalently from the effective operators in the renormalized Hilbert space $\left(\mathcal{H}^{\prime}\right)$. The renormalized Hamiltonian $\left(H^{\prime}\right)$ defined above ensures that if $\left|\psi_{0}^{\prime}\right\rangle$ is the ground state of $H^{\prime}$, $\left|\psi_{0}\right\rangle=T\left|\psi_{0}^{\prime}\right\rangle$ is the ground state of $H$ imposing $T^{\dagger} T=\mathbf{1}_{\mathcal{H}^{\prime}}$.

Next we discuss a prescription to calculate the ground-state fidelity within the QRG approach. $\underline{\underline{20}}$ In this respect, we recall the definition of the ground-state fidelity $F$, for a finite system size $N$,

$$
F \equiv F(\lambda, \delta, N)=\left\langle\psi_{0}\left(\lambda_{-}\right) \mid \psi_{0}\left(\lambda_{+}\right)\right\rangle
$$

where $\left|\psi_{0}\left(\lambda_{ \pm}\right)\right\rangle$is the normalized ground state at arbitrary coupling $\lambda_{ \pm} \equiv \lambda \pm \delta / 2$ and $\delta$ stands for a small variation of $\lambda$. According to the QRG prescription, the fidelity can be expressed in terms of the ground state of the renormalized Hamiltonian utilizing the embedding operator,

$$
\begin{aligned}
F & =\left\langle\psi_{0}^{\prime}\left(\lambda_{-}\right)\left|T^{\dagger}\left(\lambda_{-}\right) T\left(\lambda_{+}\right)\right| \psi_{0}^{\prime}\left(\lambda_{+}\right)\right\rangle \\
& =\left\langle\psi_{0}\left(\lambda_{-}^{\prime}\right)\left|T^{\dagger}\left(\lambda_{-}\right) T\left(\lambda_{+}\right)\right| \psi_{0}\left(\lambda_{+}^{\prime}\right)\right\rangle .
\end{aligned}
$$

In fact the group property of renormalization justifies that $\left|\psi_{0}^{\prime}(\lambda)\right\rangle=\left|\psi_{0}\left(\lambda^{\prime}\right)\right\rangle$ where $\lambda^{\prime}$ is the renormalized coupling. The operator product $T^{\dagger}\left(\lambda_{-}\right) T\left(\lambda_{+}\right)$establishes the renormalization of fidelity, which provides $F$ in terms of the fidelity of the renormalized ground state $\left(F^{\prime}\right)$. In the underlying model, the coupling $\lambda$ is composed of two parameters $\Delta$ and $D$, which requires to define a small deviation for both couplings, namely $\delta_{\Delta}$ and $\delta_{D}$. A straightforward calculation leads to the following expression

$$
T_{I}^{\dagger}\left(D_{-}, \Delta_{-}\right) T_{I}\left(D_{+}, \Delta_{+}\right)=\omega_{I} \mathbf{1}_{I}+v_{I}\left(S_{I}^{z}\right)^{2},
$$

where $\omega_{I}$ and $v_{I}$ are scalar functions of the coupling constants $\left(D_{-}, \Delta_{-} ; D_{+}, \Delta_{+}\right)$. Moreover, the ground states of all blocks, which are taken into account in the calculation of Eq. (7) belong to $S^{z}=0$ sector, which renders zero value for the second term in Eq. (8). Therefore, including the contribution of all blocks the renormalization of fidelity is given by

$$
F=\left(\omega_{I}\left(D_{-}, \Delta_{-} ; D_{+}, \Delta_{+}\right)\right)^{N / 3} F^{\prime}
$$

where $F^{\prime}$ is the renormalized fidelity. The procedure is iterated $m$-times associated with the size of system $N=3^{m+1}$, which connects the original fidelity $(F)$ to the fidelity of a system, which has been renormalized $m$-times $\left(F^{(m)}\right)$,

$$
F=\left(\prod_{n=0}^{m-1}\left[\omega_{I}\left(D_{-}^{(n)}, \Delta_{-}^{(n)} ; D_{+}^{(n)}, \Delta_{+}^{(n)}\right)\right]^{\frac{N}{3^{n+1}}}\right) F^{(m)} .
$$

For simplicity we calculate the ground-state fidelity at fixed $\Delta$ and two slightly different values of $D$, namely $D \pm \delta_{D} / 2$ for three different values of $\delta_{D} \equiv \delta=0.001,0.005,0.01$. Hence, we track the quantum critical points by adjusting $D$. An abrupt drop of fidelity in the vicinity of quantum critical point is a consequence of an essential change in the structure of ground state, which is usually accompanied by a divergence/maximum of fidelity susceptibility. Therefore, fidelity or its corresponding susceptibility are reliable signatures of quantum criticality. As far as the ground state is normalized to unity, the leading term of the fidelity is expressed by

$$
F(D, \delta ; \Delta, N) \simeq 1-\frac{N \delta^{2}}{2} \chi(D ; N),
$$

where $\chi$ is the fidelity susceptibility, which is defined by $\chi \equiv$ $\frac{-1}{N} \frac{\partial^{2} F}{\partial \delta^{2}}$.

We have plotted $\chi$ versus $D$ at $\Delta=0$ in Fig. 3 for different values of $\delta$ and system sizes. Accordingly, $\chi$ extinguishes the dependence on $\delta$ such that all plots with different 


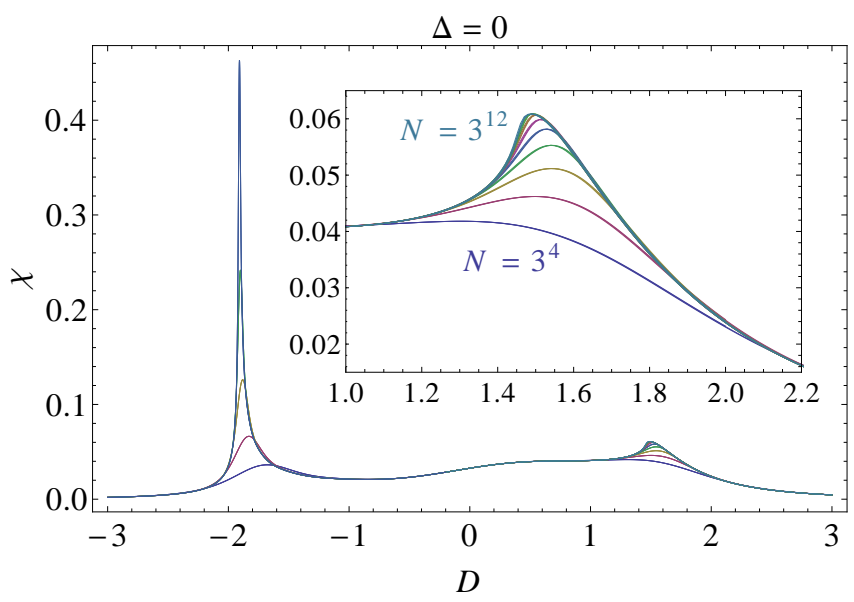

FIG. 3. (color online) Fidelity susceptibility $(\chi)$ versus $D$ at $\Delta=0$ for different sizes $N=3^{m+1}, m=3, \ldots, 11$ and $\delta=0.001,0.005,0.01$. The size dependence is only visible around the critical points presented by different colors. The left peak corresponds to the Néel-Haldane quantum critical point while the right one presents the Haldane to large- $D$ quantum phase transition. Inset: The right peak around $D \simeq 1.5$ is plotted in larger scale to clarify the behavior at the Haldane-large- $D$ quantum critical point.

$\delta=0.01,0.005,0.001$ fall on each other except at the critical points where the size dependence turns out. Close to a quantum critical point $\chi$ exhibits a peak, which intensifies by increasing the system size. However, for off-critical regions there is no size dependence on $\chi$, which is clearly visible from Fig. 3. The left peak in Fig. 3 represents the Néel to Haldane phase transition where Néel order exists for $D \lesssim-2.0$ while the right peak corresponds to the Haldane-large- $D$ quantum phase transition. The right peak around $D \sim 1.45$ is plotted in larger scale as an inset in Fig. 3 to justify its size dependence as a signature of quantum phase transition. Similarly for the isotropic case $(\Delta=1)$, the fidelity susceptibility versus $D$ is plotted in Fig 4 for the same values of $\delta=0.01,0.005,0.001$ and system sizes $N=3^{n}, n=4, \ldots, 12$. Two signatures of quantum phase transition are observed, the left sharp peak is clearly seen while the second peak appears on a smaller scale around $D \sim 1.7$. To find out the second peak we have plotted the region $0<D<3$ in larger scale as an inset in Fig. 4 . which verifies an enhancing peak around $D \sim 1.7$ that is corresponding to the Haldane-large- $D$ phase transition.

In both Figs. 3 and 4 the left peak in $\chi$ is higher and sharper than the right one. The reason why the two transition show such a drastically different behavior is not completely clear. One might speculate that the reason is inherited to the two different mechanism for the Néel-Haldane and Haldane-large- $D$ phase transitions. The former is associated with a spontaneous symmetry breaking, which sets up a nonzero staggered magnetization in the Néel phase while the latter corresponds to a quantum phase transition to a symmetry protected topological phase. However, the QRG approach to fidelity is able to detect both types of phase transition as witnessed above.

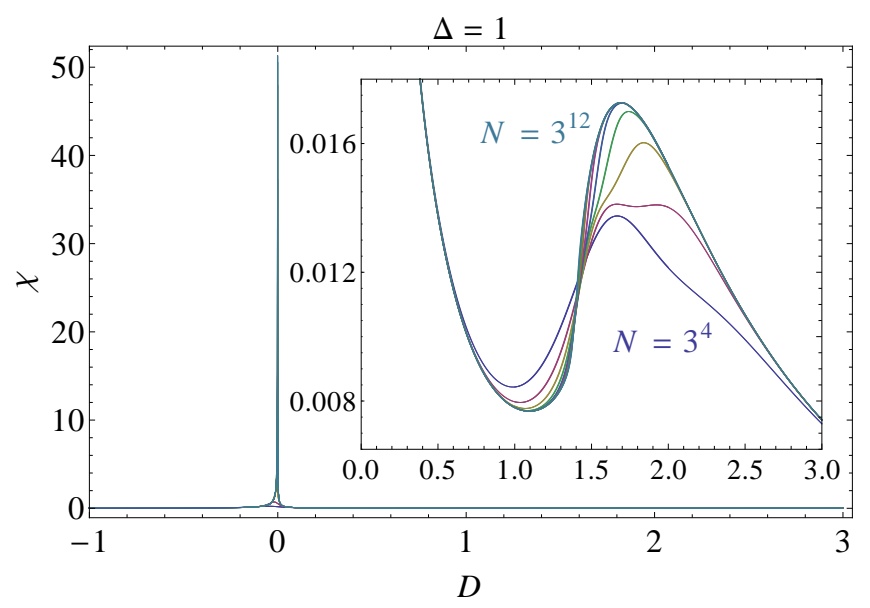

FIG. 4. (color online) Fidelity susceptibility $(\chi)$ versus $D$ at the isotropic point $(\Delta=1)$ for different sizes $N=3^{m+1}, m=$ $3, \ldots, 11$ and $\delta=0.001,0.005,0.01$. The very sharp peak at $D \simeq 0$, which is associated to the Néel-Haldane quantum critical point induces a scale, which interdicts another peak at $D \sim 1.7$. Inset: $\chi$ vs. $D$ for $0<D<3$ in larger scale such that the peak on Haldane-large- $D$ phase transition is clearly observable.

As described above, the QRG fidelity approach can be used to find the quantum phase boundary of our model. Moreover, the analysis of the QRG-flow Eq. (5) gives a clear picture of the topography of ground state phase diagram as depicted in Fig. 5] The QRG-flow has two types of fixed points, those which are attractive in all directions stand for stable phases and those which are repulsive at least in one direction represent quantum critical points. The fixed points $\left(D^{*}, \Delta^{*}\right)$ are denoted by $\circledast$ in Fig. [5] and are labeled $\mathrm{P}_{1}:(0.0,1.0)$, $\mathrm{P}_{2}:(0.58,0.0), \mathrm{P}_{3}:(1.45,0.0), \mathrm{P}_{4}:(-2.0,0.0), \mathrm{P}_{5}:(2.5,3.0)$ and two others for extremely large couplings, namely $(\infty, 0.0)$ and $(-\infty, \infty) \cdot \mathrm{P}_{1}$ is associated to the isotropic $S=1$ Heisenberg model (without single ion anisotropy) which retains the SU(2) symmetry upon QRG transformation and belongs to the Haldane phase. The $\mathrm{SU}(2)$ symmetric fixed point $\left(\mathrm{P}_{1}\right)$ is unstable upon adding axial anisotropy either by easy-axis or singleion types. The fixed point $\mathrm{P}_{1}$ is isotropic, i.e., it has the full $\mathrm{SU}(2)$ symmetry, while $\mathrm{P}_{2}$ has a $U(1) \times Z 2$ symmetry. The black line, which passes through $\mathrm{P}_{1}$ specifies the phase boundary between the Néel and Haldane phases. All couplings in the closed bounded region labeled "Haldane" run to the stable fixed point $\mathrm{P}_{2}$ under $\mathrm{QRG}$ transformations while $\mathrm{P}_{3}$ is unstable in vertical direction and corresponds to the critical point between Haldane and large- $D$ phases on the $\Delta=0$ line. The black line which originates from $\mathrm{P}_{5}$ and ends at $\mathrm{P}_{3}$ represents the border between Haldane and large- $D$ phases as a function of $\Delta$. On the negative part of vertical axis the unstable fixed point $\mathrm{P}_{4}$ stands for the critical point between Néel and Haldane phases. The tri-critical point $\mathrm{P}_{5}$ is associated to the point where the two borders between Néel-Haldane and Haldanelarge- $D$ merge and the model belongs to either Néel or large$D$ phases.

We showed that using the QRG approach to the groundstate fidelity, we can obtain the right topology of the phase 


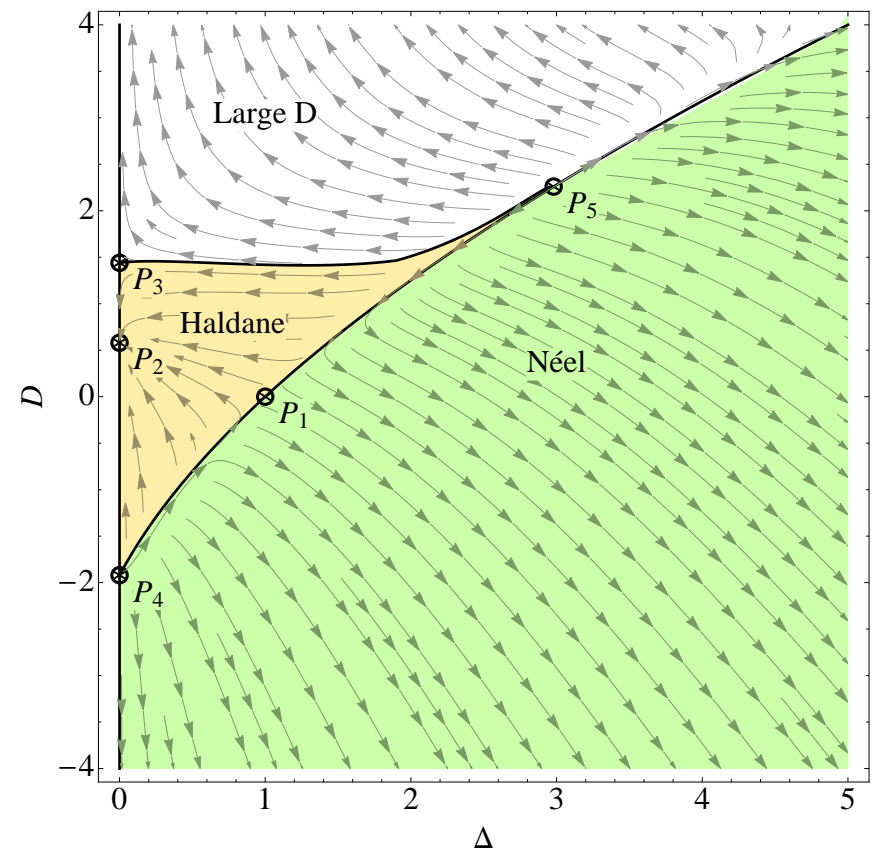

FIG. 5. (color online) The QRG-flow, which specifies the phase diagram of $S=1 \mathrm{XXZ}$ model with single ion anisotropy (D) where $\Delta$ stands for easy-axis anisotropy coupling. The critical boundaries are denoted by black lines and $\circledast$ represent the fixed points $P_{1}-P_{5}$. The phase diagram is composed of Néel, Haldane and large- $D$ phases.

diagram. However, the phase boundaries are shifted compared to the known (numerically exact) results $\underline{22}$

\section{EXACT DIAGONALIZATION}

In the following, the ground-state fidelity of the Hamiltonian defined in Eq. (1) is calculated exactly for finite sizes using the Lanczos method. Here, we compute the fidelity for two ground states with slightly different values of $D$ at fixed $\Delta$, i.e.

$$
F=\left\langle\psi_{0}(\Delta, D-\delta / 2) \mid \psi_{0}(\Delta, D+\delta / 2)\right\rangle,
$$

where $\delta=0.01$. We have plotted fidelity versus $D$ in Fig. 6 for $\Delta=0$ and even number of sites on periodic chain $N=$ $8,10,12,14$. The fidelity has two minima at $D \sim-2$ and $D \sim 1.5$ where clear finite size effects are observed, which are signatures of quantum phase transition. Moreover, we plot the fidelity susceptibility $(\chi)$ versus $D$ as inset in Fig. 6 where two peaks associated to the minima of fidelity confirm the existence of the quantum critical points. The peak of susceptibility has an apparent maximum where its corresponding height $\left(\chi_{m}\right)$ scales like $\chi_{m} \sim N^{\alpha}$. For the inset of Fig. 6 the left peak scales linearly with system size $(N)$ and the right peak, which corresponds to the Haldane-large- $D$ phase transition scales as $N^{0.63}$. A similar analysis for $\Delta=1$ shows that the left peak of $\chi$ around $D \simeq 0$ scales with $N^{0.807}$ and

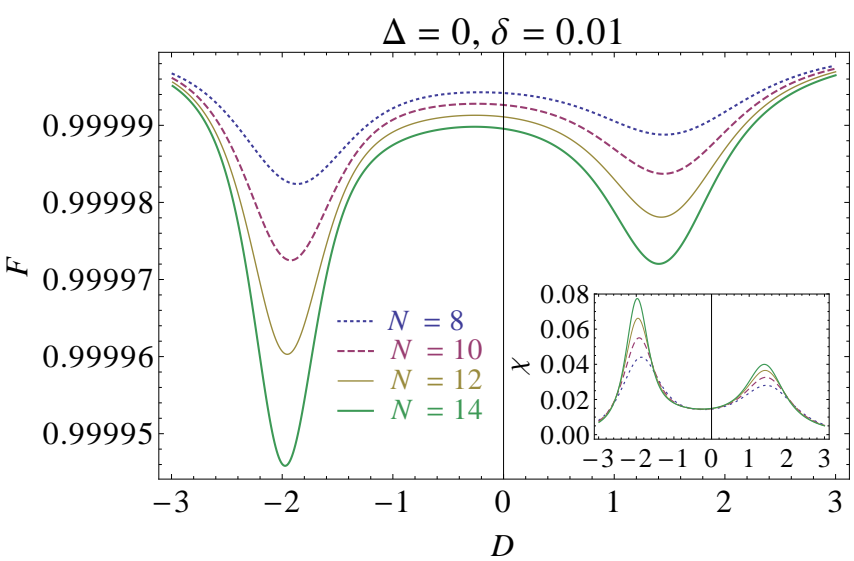

FIG. 6. (color online) Exact diagonalization results of fidelity (F) versus $D$ for $\Delta=0, \delta=0.01$ and on chain lengths $N=$ $8,10,12,14$. The corresponding fidelity susceptibility $(\chi)$ is plotted as an inset.

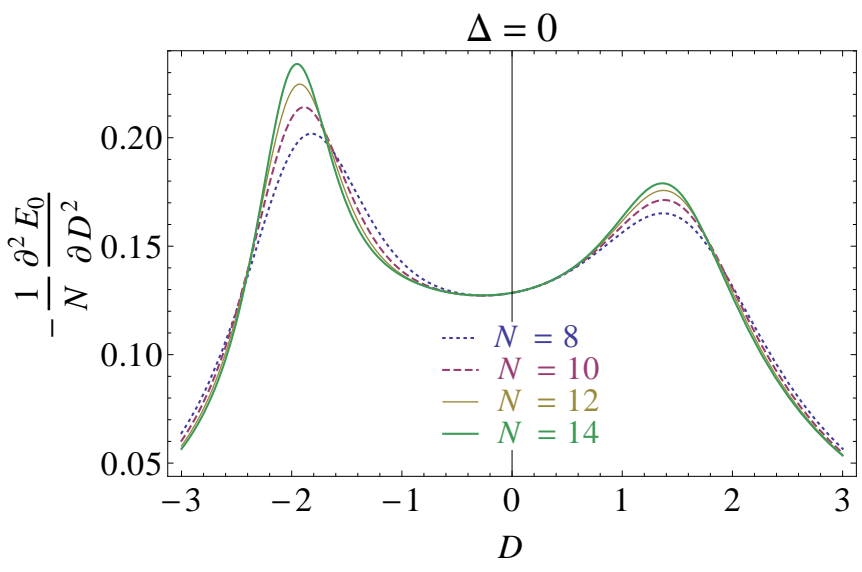

FIG. 7. (color online) Second derivative of ground state energy density versus $D$ for $\Delta=0$.

for the right one corresponding to Haldane-large- $D$ transition $\chi_{m} \sim N^{0.561}$. In all cases the fidelity susceptibility diverges in the thermodynamic limit although its divergence is linear only for the Néel-Haldane phase transition at $\Delta=0$ and is sub-linear for other cases specially for the Haldane to large- $D$ phase transition.

According to Ref. 27, a quantum phase transition does not always lead to a superextensive growth of fidelity susceptibility which means that $\chi$ can be even finite as $N \rightarrow \infty$ for gapless systems. However, our analysis on small system sizes $(N \leq 14)$ shows that the fidelity susceptibility is always divergent both on the Néel-Haldane and Haldane-large- $D$ phase transitions. Accordingly, we would expect to observe similar peaks in the 2 nd derivative of ground state energy density. We have plotted $\frac{-1}{N} \frac{\partial^{2} E_{0}}{\partial D^{2}}$ versus $D$ for $\Delta=0$ in Fig. 7 where $E_{0}$ is the ground state energy of a chain of $N$ spins within periodic boundary condition. As is expected the peaks in susceptibility (inset of Fig. 6) are sharper than the corresponding 
ones in the 2nd derivative of energy close to quantum critical points manifesting that $\chi \sim \frac{1}{E_{g a p}^{2}}$ while $\frac{\partial^{2} E_{0}}{\partial D^{2}} \sim \frac{1}{E_{g a p}}$ where $E_{g a p}$ is the energy gap. Although the results of exact diagonalization shows a divergent behavior of $\chi$ and clear size dependent maximum on the 2 nd derivative of energy density for $\Delta=0$, the position of peak $D_{m}$ for different sizes does not satisfy the scaling relation $\left|D_{m}(N)-D_{c}\right| \sim N^{-1 / \nu}$ where $D_{c}$ is the quantum critical value and $\nu$ is the exponent which shows the divergence of correlation length. ${ }^{27}$ Therefore, the accurate determination of the quantum critical point should be obtained from the specific property of the model, which will be discussed in next subsection.

To get more insight on the fidelity behavior of the whole phase diagram we have computed the fidelity for all parameters in the range of $-3 \leq D \leq+3$ and $0 \leq \Delta \leq 2$. The exact diagonalization is performed on a periodic chain of $N=14$ and the fidelity is calculated at fixed $\Delta$ for two ground states with slightly different $D$ values, namely $D-\delta / 2$ and $D+\delta / 2$ with $\delta=0.01$. We present the three-dimensional plot of fidelity susceptibility versus $D-\Delta$ plane in Fig. 8-(top). The grid points on the $\Delta$ axis is also 0.01 . A clear set of peaks similar to the inset of Fig. 6is observed in Fig. 8-(top) which is the signature of quantum phase transition. The left set of peaks, which is stronger than the right one is a representative of Néel to Haldane phase transition while the right set of peaks corresponds to the Haldane to large- $D$ phase boundary. We also show the contour plot of the peaks in Fig 8 -(bottom) at different scale to show the boundary more clearly. The three dimensional plot of $\chi$ is consistent with the quantum phase diagram of Fig 5 confirming that ground-state fidelity is a good indicator to find quantum phase transition.

\section{A. Parity order parameter}

Here we discuss how the bond inversion symmetry of the Hamiltonian can be used to determine the phase transition from the Haldane phase to the large- $D$ phase very accurately from exact diagonalization studies. In Refs. 7 and 8 it was shown that the $S=1$ Haldane phase is protected by the link inversion symmetry. A simple picture helps to realize the nature of the two phases: Let us suppose that the Haldane phase is described by the valence bond solid (VBS) configuration where each spin $S=1$ is composed of two $S=1 / 2$ spins and the ground state is a sequence of singlets formed between two neighboring $S=1 / 2$ spins. We now consider an odd number of $S=1$ spins on a periodic chain and invert the lattice about its reflection symmetric plane (see Fig. 2 of Ref.7). The ground state of the Haldane phase gets a $\pi$-phase under inversion as a result of inverting an odd number of singlet bonds. However, the large- $D$ phase is totally symmetric under reflection, which returns a phase of 0 . Therefore the ground states are in different symmetry sectors in the two phases and a level crossing occurs at the critical point (i.e., the ground state in the Haldane phase is in the sector, which is odd under inversion and the large- $D$ phase in the sector, which is even). This
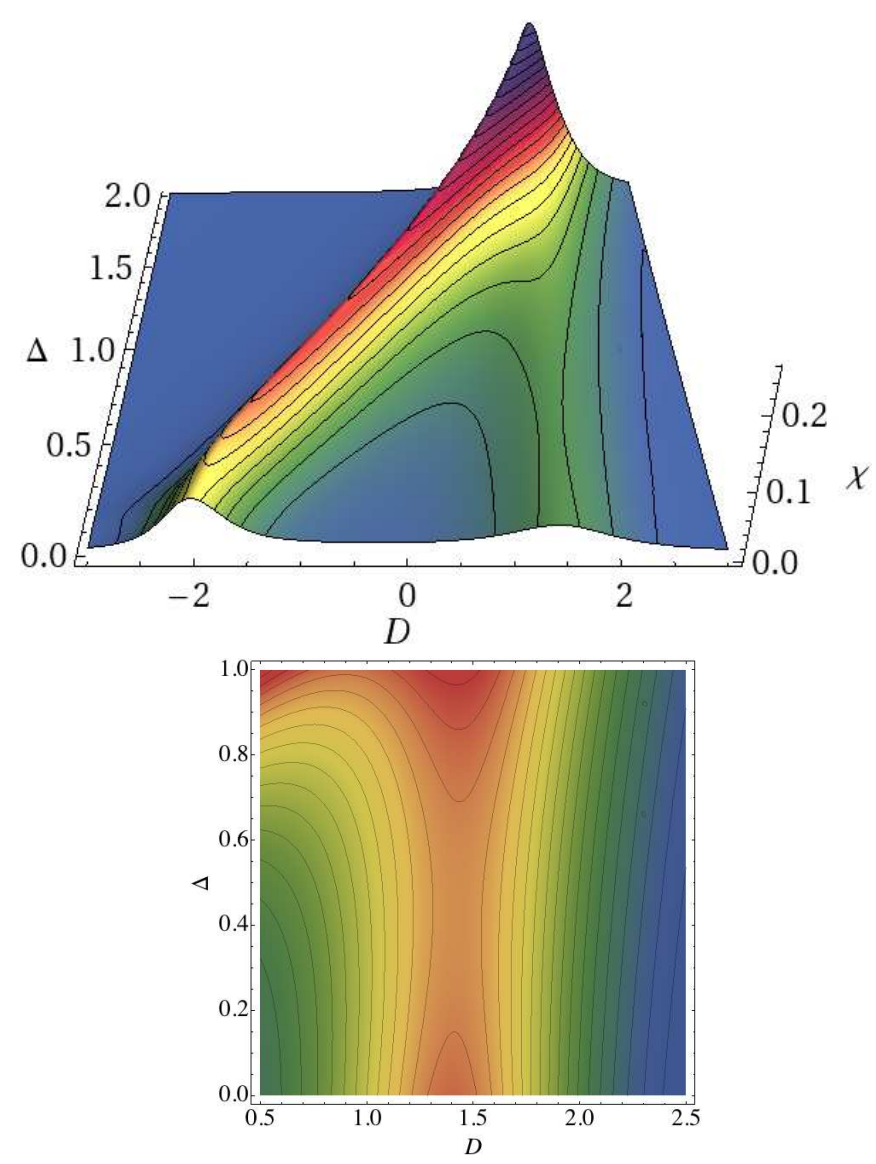

FIG. 8. (color online) Top: The 3D-plot of fidelity susceptibility $(\chi)$ versus $D-\Delta$ plane. The peaks of $\chi$ correspond to the critical phase boundaries where the higher peaks represent the border between Néel-Haldane phases and the lower peaks is a representative for the Haldane to large- $D$ quantum phase transition. Bottom: The contour plot of Haldane to large- $D$ transition in larger scale for more clear vision.

distinction has been used in exact diagonalization of $S=2$ chains to detect symmetry protected phases $\frac{35}{3}$ In rings with an even number of sites, however, the ground state is always in the even sector and thus we do not expect a level crossing. In both cases, it is still possible to distinguish the phases by calculating the expectation value of an operator, which inverts a block of consecutive spins $\frac{36}{2}$ This order parameter can been seen as a generalization of the string order parameter introduced in Ref. 37.

For the implementation of the inversion symmetry based order parameter, we define permutation operators $\mathcal{P}_{i, j}$, which exchange the positions of two spins $i$ and $j$ :

$$
\mathcal{P}_{i, j}=\vec{S}_{i} \cdot \vec{S}_{j}+\left(\vec{S}_{i} \cdot \vec{S}_{j}\right)^{2}-\mathbf{1},
$$

where $\vec{S}_{i}$ is the spin-1 operator at site $i$ and $\mathbf{1}$ is the identity operator.

We begin by considering the case of a ring with an odd number of spins. The expectation value of different permutations of the $N=15$ ring versus $D$ is shown in Fig. 9 The expectation value is always negative in the Haldane phase 


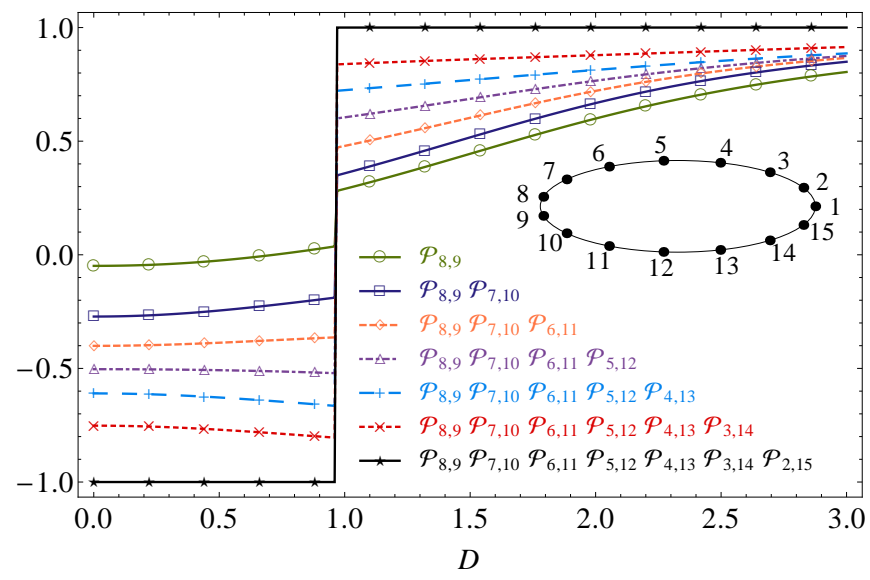

FIG. 9. (color online) The ground state expectation value of different permutation operators versus $D$ on $N=15$ (odd-N) isotropic spin $S=1$ chain with periodic boundary conditions. The Haldane phase returns negative value while it jumps to a positive value for the large$D$ phase.

TABLE I. (color online) The critical point $\left(D_{c}\right)$ between the Haldane and large- $D$ phase for different values of anisotropies $(\Delta)$.

\begin{tabular}{|c||c|c|c|c|c|c|c|c|}
\hline$\Delta$ & 0 & 0.1 & 0.2 & 0.3 & 0.4 & 0.5 & 0.6 & 0.7 \\
\hline$D_{c}$ & 0.347 & 0.403 & 0.458 & 0.515 & 0.575 & 0.636 & 0.698 & 0.763 \\
\hline \hline$\Delta$ & 0.8 & 0.9 & 1 & 1.1 & 1.2 & 1.3 & 1.4 & 1.5 \\
\hline$D_{c}$ & 0.830 & 0.898 & 0.968 & 1.039 & 1.113 & 1.187 & 1.265 & 1.343 \\
\hline
\end{tabular}

and jumps to a positive value upon arriving in the large- $D$ phase, clearly distinguishing the two phases. The reflection symmetry, which is expressed by a two-point permutation, $P_{r}=\mathcal{P}_{2,15} \mathcal{P}_{3,14} \mathcal{P}_{4,13} \mathcal{P}_{5,12} \mathcal{P}_{6,11} \mathcal{P}_{7,10} \mathcal{P}_{8,9}$ returns -1 in the Haldane phase and +1 for the large- $D$ one. We have calculated $\left\langle\psi_{0}\left|P_{r}\right| \psi_{0}\right\rangle$ on periodic chain with $N=9,11,13,15$ and found the value of $D_{p}$ at which it changes sign form - 1 to +1 . The finite size analysis of our data by extrapolating with $D_{p}=D_{c}+a N^{-b}$ gives the critical point $\left(D_{c}\right)$ in the thermodynamic limit. We have presented the critical point between the Haldane and large- $D$ phases within 3-digits of accuracy in Table 1. A comparison with recent large scale DMRG results 28 for $\Delta=0.5$ and 1 shows a perfect agreement up to the $3 \mathrm{rd}$ digit. This is remarkable as our results are obtained from exact diagonalization on small clusters, which require much less resources.

Next we examine the ground state expectation value of the permutation operators on an even- $\mathrm{N}$ chains with periodic boundary condition. We have plotted the permutation expectation value of $\mathrm{N}=14$ versus $D$ in Fig. 10. The expectation value still changes its sign when going from the Haldane phase into the large D phase, however, finite size effects are much stronger in this case and an accurate determination of the critical point is not possible. As expected, the order parameter does not show a jump and we obtain always +1 when invert-

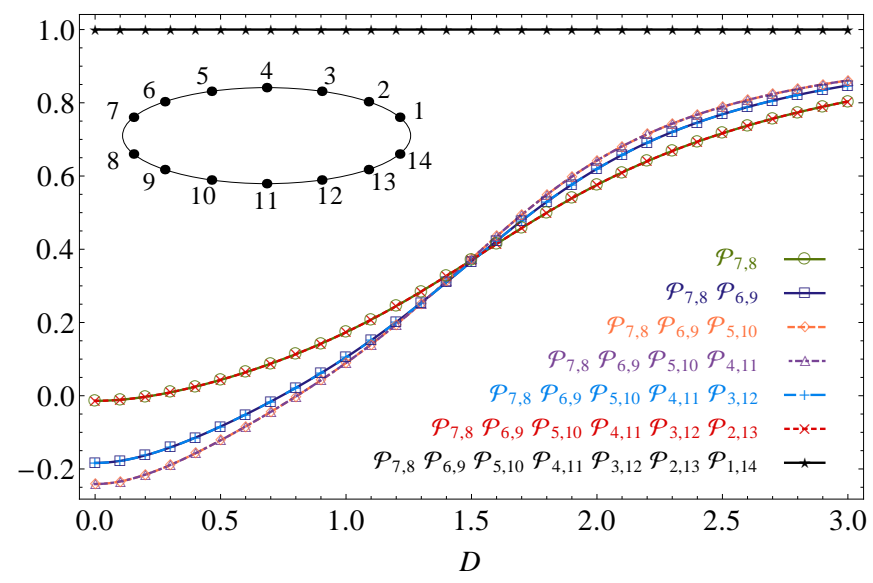

FIG. 10. (color online) The ground state expectation value of different permutation operators versus $D$ on $\mathrm{N}=14$ (even-N) isotropic spin $S=1$ chain with periodic boundary conditions. The Haldane phase returns negative value while it gets positive continuously for the large- $D$ phase.

ing the full chain, i.e., the ground state is always in the even sector.

\section{SUMMARY AND CONCLUSIONS}

We have studied the anisotropic spin-1 Heisenberg chain with single ion anisotropy by utilizing quantum renormalization group and exact diagonalization methods. We have applied the recent implementation of QRG to calculate the ground-state fidelity ${ }^{20}$ of the spin-1 chain without the need to calculate the ground state exactly. We have obtained the renormalization of fidelity as an analytic expression in terms of the renormalized coupling constants for the whole phase diagram. The fidelity shows a drop at the quantum critical point irrespective of being due to global symmetry breaking like Néel-Haldane transition or a symmetry protected topological phase transition like Haldane-large- $D$ transition. It confirms that fidelity is an appropriate candidate to discriminate the quantum phase transitions even if it is a topological one. We have also obtained the fidelity susceptibility, which shows a growing peak at the quantum critical point by increasing size of system where its qualitative behavior does not depend on the easy axis anisotropy. The growing peak is divergent at the Néel-Haldane critical point while it is just a maximum at the Haldane-large- $D$ phase transition. Although the quantitative results of QRG fidelity is not accurate, its qualitative description of the phase diagram is fairly good in addition to the RG-flow which gives a topography of the phase diagram.

We have also analyzed the fidelity and its susceptibility data, which come from exact diagonalization simulation on finite periodic chains $(N=8, \ldots, 15)$. Two very different types of behaviors have been observed for even and odd number of sites. For even number of sites, $N=8,10,12,14$, the fidelity shows a drop at the quantum critical points and its cor- 
responding susceptibility presents a peak. The susceptibility peak is sharp and divergent at the Néel-Haldane critical point, which grows almost linearly with $N$ while the corresponding peak at the Haldane-large- $D$ transition is wide and grows sub-linearly with $N$. This is in agreement with the scaling argument presented in Ref. 27, which states that the quantum phase transition does not always lead to a superextensive growth of fidelity susceptibility. In contrast to Ref. 26 our exact diagonalization results show similar qualitative behavior for all easy axis anisotropies, none of our results fall into the scaling behavior $\left|D_{m}(N)-D_{c}\right| \sim N^{-1 / \nu}$, which suggests two facts: (i) The topological Haldane-large- $D$ phase transition has strong non-local quantum correlations which requires an intensive numerical simulation ${ }^{28}$ for a very large system size to get the quantum critical point accurately. (ii) The scaling behavior of fidelity could be different at small size limit $N \delta<1$ and large size one $N \delta>1$, similar to what has been observed for the Ising chain in transverse field. ${ }^{20,38}$

The exact diagonalization data of clusters with an odd number of sites $(N=9,11,13,15)$ unveils a symmetry, which protects the topological Haldane-large- $D$ transition: The parity "string order parameter" in Fig. 9 shows an abrupt change of parity at the topological phase boundary. This is a very accurate signature of the Haldane-large- $D$ phase transition on small chains, which gives the quantum critical point accurately as obtained in Table. [1 It suggests an investigation of a similar quantity to identify other types of topological phase transitions. It has to be noticed that the expectation value of inversion operator $\left(\left\langle P_{r}\right\rangle\right)$ is a non-local quantity and thus expected be sensitive for a topological phase transition. However, the expectation value of a single permutation operator like $\left\langle P_{8,9}\right\rangle$ (for $N=15$ ) encounters a sign change at the phase boundary, which can be considered as a signature of the mentioned phase transition. This type of order parameters is thus helpful to determine the phase boundary of topological transition on small size clusters, which need less resources.

\section{ACKNOWLEDGMENTS}

This work was supported in part by the Office of VicePresident for Research of Sharif University of Technology. A.
L. acknowledges the support from the Alexander von Humboldt Foundation.

\section{Appendix A: The QRG procedure}

The renormalized Hamiltonian $H^{\prime}(\mathrm{Eq} 2)$ is obtained within QRG procedure using the embedding operator, Eq, 4, which is given by

$$
H^{\prime}=\sum_{I=1}^{N / 3}\left(T_{I}^{\dagger} h_{I}^{B} T_{I}+T_{I}^{\dagger} T_{I+1}^{\dagger} h_{I, I+1}^{B B} T_{I+1} T_{I}\right)
$$

The first part of this projections leads to

$$
T_{I}^{\dagger} h_{I}^{B} T_{I}=E_{0} \mathbf{1}+\left(E_{1}-E_{0}\right)\left(S_{I}^{z}\right)^{2},
$$

where $E_{0}$ and $E_{1}$ are the two lowest eigenvalues of the block Hamiltonian, namely $E_{0}$ is the smallest root of the following equation,

$$
E^{3}+E^{2}(\Delta-4 D)+E\left(4 D^{2}-2 \Delta D-6\right)+8 D=0,
$$

and $E_{1}$ is the smallest solution of

$$
\begin{aligned}
& E^{4}+E^{3}(2 \Delta-8 D)+E^{2}\left(22 D^{2}-10 \Delta D-5\right) \\
& +E\left(-24 D^{3}+14 \Delta D^{2}+24 D-6 \Delta\right) \\
& +9 D^{4}-6 \Delta D^{3}-27 D^{2}+14 \Delta D=0 .
\end{aligned}
$$

The second term of Eq A1 defines the effective interaction between blocks $I$ and $I+1$ in terms of renormalized operators

$$
\begin{aligned}
& T_{I}^{\dagger} S_{I, j}^{\alpha} T_{I}=X_{\mathrm{ren}} S_{I}^{\prime \alpha} ; j=1,3 ; \alpha=x, y, \\
& T_{I}^{\dagger} S_{I, j}^{z} T_{I}=Z_{\mathrm{ren}} S_{I}^{\prime z} ; j=1,3,
\end{aligned}
$$

where $S_{I, j}^{\alpha}$ represents the $\alpha$ component of spin- 1 at site- $j$ in the $I$-th block of the original Hamiltonian and $S_{I}^{\prime \alpha}$ is spin-1 operator defined for the $I$-th block in the renormalized Hilbert space. The renormalization coefficients $X_{\text {ren }}$ and $Z_{\text {ren }}$ are given by the following expressions,

$$
\begin{aligned}
X_{\text {ren }}= & \frac{1}{\sqrt{A_{5} A_{9}}}\left[2\left(E_{0}-2 D\right)+2\left(E_{1}-3 D\right)\left[4 D^{2}-2 D\left(\Delta+2 E_{0}\right)+E_{0}\left(\Delta+E_{0}\right)-2\right]-\right. \\
& A_{2} A_{3}\left(D-E_{1}\right)\left[4 D^{2} E_{0}-2 A_{8}-2 D\left[E_{0}\left(\Delta+2 E_{0}\right)-3\right]+E_{0}^{2}\left(\Delta+E_{0}\right)-6 E_{0}\right]+ \\
& \left.2 A_{1} A_{2} A_{3}\left(A_{8}-D+E_{0}\right)-\frac{A_{7}\left[A_{1} A_{2} A_{3}\left(D-E_{1}\right)-6 D+2 E_{1}\right]}{2 D-E_{0}}\right], \\
Z_{\text {ren }}= & \frac{1}{A_{5}}\left[A_{3}^{2} A_{2}^{2}\left(D-E_{1}\right)^{2}-\left[\left(D-E_{1}\right)\left[A_{1} A_{2} A_{3}\left(D-E_{1}\right)+4 E_{1}-12 D\right]+2\right]^{2}+4\left(E_{1}-3 D\right)^{2}+4\right] .
\end{aligned}
$$


We have defined the following relations for th $A_{i}$ constants,

$$
\begin{aligned}
A_{1}= & 2 \Delta-3 D+E_{1}, \\
A_{2}= & E_{1}^{2}-4 D E_{1}+3 D^{2}-1, \\
A_{3}= & \frac{1}{\Delta-2 D+E_{1}}, \\
A_{4}= & \frac{1}{\Delta-2 D+E_{0}}, \\
A_{5}= & A_{3}^{2}\left[A_{1} A_{2}\left(D-E_{1}\right)+2\left(3 D-E_{1}\right)\left(2 D-\Delta-E_{1}\right)\right]^{2} \\
& +A_{2}^{2} A_{3}^{2}\left(D-E_{1}\right)^{2}+A_{1}^{2} A_{2}^{2} A_{3}^{2}+4\left(E_{1}-3 D\right)^{2}+4+ \\
& {\left[\left(D-E_{1}\right)\left[A_{1} A_{2} A_{3}\left(D-E_{1}\right)+4\left(E_{1}-3 D\right)\right]+2\right]^{2}, } \\
A_{6}= & 16 D^{4} E_{0}-8 D^{3}\left[2 E_{0}\left(\Delta+2 E_{0}\right)-3\right] \\
& +4 D^{2}\left[6 E_{0}^{3}+6 \Delta E_{0}^{2}+\left(\Delta^{2}-12\right) E_{0}-3 \Delta\right] \\
& -2 D\left[4 E_{0}^{4}+6 \Delta E_{0}^{3}+\left(2 \Delta^{2}-15\right) E_{0}^{2}-9 \Delta E_{0}+4\right] \\
& +E_{0}^{2}\left(\Delta+E_{0}\right)\left[E_{0}\left(\Delta+E_{0}\right)-6\right]+6 E_{0},
\end{aligned}
$$

$$
\begin{aligned}
A_{7}= & -E_{0}^{3}+E_{0}^{2}(4 D-\Delta)+2 E_{0}\left(D \Delta-2 D^{2}\right)-4 D+2, \\
A_{8}= & \frac{A_{4}\left(3 E_{0}-4 D\right)}{2 D-E_{0}}, \\
A_{9}= & \frac{A_{4}^{2} A_{6}^{2}+A_{7}^{2}}{\left(E_{0}-2 D\right)^{2}}+2\left(E_{0}-2 D\right)^{2} \\
& +4\left(A_{8}-D+E_{0}\right)^{2}+4 \\
& +\left[4 D^{2}-2 D\left(\Delta+2 E_{0}\right)+E_{0}\left(\Delta+E_{0}\right)-2\right]^{2} .(\mathrm{A} 7)
\end{aligned}
$$

Finally, the renormalized coupling constants, Eq5 are given by the following relations,

$$
\begin{aligned}
J^{\prime} & =\left(X_{\text {ren }}\right)^{2} J, \\
\Delta^{\prime} & =\left(\frac{Z_{\text {ren }}}{X_{\text {ren }}}\right)^{2} \Delta, \\
D^{\prime} & =\frac{E_{1}-E_{0}}{\left(X_{\text {ren }}\right)^{2}} .
\end{aligned}
$$

* langari@ sharif.edu, http://sharif.edu/ langari/

1 S. Sachdev, Quantum Phase Transitions (Cambridge University Press, 2001).

2 L. D. Landau, Phys. Z. Sowjetunion 11, 26 (1937).

${ }^{3}$ F. D. M. Haldane, Phys. Rev. Lett. 50, 1153 (1983).

${ }^{4}$ F. D. M. Haldane, Phys. Lett. 93A, 464 (1983).

5 X. Chen, Z.-C. Gu, and X.-G. Wen, Phys. Rev. B 83, 035107 (2011)

$6 \mathrm{X}$. Chen, Z.-C. Gu, and X.-G. Wen, Phys. Rev. B 84, 235128 (2011)

7 F. Pollmann, E. Berg, A. M. Turner, and M. Oshikawa, Phys. Rev. B 85, 075125 (2012).

8 F. Pollmann, A. M. Turner, E. Berg, and M. Oshikawa, Phys. Rev. B 81, 064439 (2010).

9 N. Schuch, D. Pérez-García, and I. Cirac, Phys. Rev. B 84, 165139 (2011)

10 L. Amico, R. Fazio, A. Osterloh, and V. Vedral, Rev. Mod. Phys. 80, 517 (2008).

11 L. Amico and R. Fazio, Journal of Physics A: Mathematical and Theoretictent42, Jordeoph(20209) 11, 3145 (1996)

${ }_{12}$ P. Zanardi and N. Paunković, Phys. Rev. E 74, 031123 (2006) $\quad{ }^{31}$ G. Sierra $\quad$ and

$13 \mathrm{H}-\mathrm{Q}$ Z Z J P Journal of Physics A: Mathematical and Theoretical 41, 412001 (2008) Lecture notes in physics, Vol. 478 (Springer, 1997).

14 S.-J. Gu, International Journal of Modern Physics B 24, 4371 (2010).

15 M. Kargarian, R. Jafari, and A. Langari, Phys. Rev. A 76, 060304 (2007)

16 M. Kargarian, R. Jafari, and A. Langari, Phys. Rev. A 77, 032346 (2008)

17 M. Kargarian, R. Jafari, and A. Langari, Phys. Rev. A 79, 042319 (2009)

18 K. G. Wilson, Rev. Mod. Phys. 47, 773 (1975)

19 P. Pfeuty, R. Jullian, and K. L. Penson (Springer-Verlag, 1982).
${ }^{20}$ A. Langari and A. Rezakhani, New Journal of Physics 14, 053014 (2012)

21 N. Amiri and A. Langari, Phys. Status Solidi B 250, 537 (2013).

${ }^{22}$ W. Chen, K. Hida, and B. C. Sanctuary, Phys. Rev. B 67, 104401 (2003)

23 R. Botet and R. Jullien, Phys. Rev. B 27, 613 (1983)

${ }^{24}$ H. J. Schulz, Phys. Rev. B 34, 6372 (1986)

25 A. Kitazawa, K. Nomura, and K. Okamoto, Phys. Rev. Lett. 76, 4038 (1996)

26 Y.-C. Tzeng, H.-H. Hung, Y.-C. Chen, and M.-F. Yang, Phys. Rev. A 77, 062321 (2008)

27 L. Campos Venuti and P. Zanardi, 28 Phys. Rev. Lett. 99, 095701 (2007) and L. Yu, Phys. Rev. B 84, 220402 (2011)

29 Y.-C. Tzeng and M.-F. Yang, Phys. Rev. A 77, 012311 (2008)

$30 \mathrm{M}$. A. Martin-Delgado and G. Sierra, G. Sierra and M. Martin-Delgado,

32 A. Langari, Phys. Rev. B 58, 14467 (1998)

33 R. Jafari and A. Langari,Physica A: Statistical Mechanics and its Applications ?

${ }^{34}$ R. Jafari and A. Langari, Phys. Rev. B 76, 014412 (2007).

35 T. Tonegawa, K. Okamoto, H. Nakano, T. Sakai, K. Nomura, and M. Kaburagi, J. Phys. Soc. 80, 043001 (2011)

36 F. Pollmann and A. M. Turner, Phys. Rev. B 86, 125441 (2012)

${ }^{37}$ M. den Nijs and K. Rommelse, Phys. Rev. B 40, 4709 (1989).

38 M. M. Rams and B. Damski, Phys. Rev. Lett. 106, 055701 (2011) 